Pacific Journal of Mathematics

INFINITE SEMIGROUPS WHOSE NON-TRIVIA 


\title{
INFINITE SEMIGROUPS WHOSE NONTRIVIAL HOMOMORPHS ARE ALL ISOMORPHIC
}

\author{
BRUCE A. Jensen
}

An infinite semigroup $S$ such that every nontrivial homomorph of it is isomorphic to $S$ is called an $H I$ semigroup. Every commutative $H I$ semigroup is a group and thus it is isomorphic to the group $Z(p)^{\infty}$, for some prime $P$. An infinite Brandt semigroup is $H I$ if and only if it has a trivial structure group. An inverse $H I$ semigroup containing a primitive idempotent is either Brandt or else it is isomorphic to a trasfinite chain of extensions of a Brandt semigroup $K$ by isomorphic copies of $K$ (where $K$ has the trivial group as its structure group). Necessary and sufficient conditions are given for a semigroup of the latter type to yield an $H I$ semigroup and an example is constructed.

In his monograph Infinite Abelian Groups, I. Kaplansky includes as exercises the following results concerning an infinite abelian group $G$ :

(1) If every subgroup of $G$ is isomorphic to $G, G$ is cyclic.

(2) If every subgroup of $G$ is finite, $G$ is isomorphic to the group $Z\left(p^{\infty}\right)$ for some prime $p$.

(3) If every proper homomorph of $G$ is finite, $G$ is cyclic.

(4) If every nontrivial homomorph of $G$ is isomorphic to $G, G$ is isomorphic to the group $Z\left(p^{\infty}\right)$ for some prime $p$.

In generalizing these results to semigroups, (1) can easily be disposed of. Suppose $S$ is a semigroup such that every subsemigroup of $S$ is isomorphic to $S$. It is clear that $S$ must be cyclic, say $S=\left\{a, a^{2}, \cdots\right\}$. However, $T=\left\{a^{2}, a^{3}, \cdots\right\}$ is a noncyclic subsemigroup of $S$ and thus $T$ is not isomorphic to $S$, a contradiction.

In [3], Jensen and Miller prove that any infinite semigroup $S$ such that every subsemigroup of $S$ is finite is a group. Thus in particular, if $S$ is commutative, $S$ is isomorphic to $Z\left(p^{\infty}\right)$ for some prime $p$.

Defining an $H F$ semigroup to be an infinite semigroup with the property that every proper homomorph is finite, it is shown in [3] that a commutative semigroup $S$ containing at least three elements is an $H F$ semigroup if and only if $S$ can be (isomorphically) imbedded in an infinite cyclic group with zero adjoined. In [2] the structure of $H F$ inverse semigroups is investigated. The structure of all $H F$ inverse semigroups that contain a primitive idempotent is determined up to the determination of all $H F$ groups. The author is unaware of any general results concerning nonabelian $H F$ groups although 
obviously some exist, e.g., any infinite simple group.

Throughout this paper, $E_{S}$ denotes the set of idempotents of the semigroup $S$ and $A^{*}$ denotes the set of nonzero elements of any $A \subseteq S$. If $S$ is a Brandt semigroup with structure group $G$ and index set $\Lambda$, we write $S=B(G ; A)$ and we denote the elements of $S^{*}$ by $\{(i, g, j) \mid i, j \in \Lambda, g \in G\}$. Then

$$
(i, g, j)\left(i^{\prime}, g^{\prime}, j^{\prime}\right)= \begin{cases}\left(i, g g^{\prime}, g^{\prime}\right) & \text { if } j=i^{\prime}, \\ 0 & \text { otherwise } .\end{cases}
$$

If $I$ is an ideal of the semigroup $S$, we identify $(S / I)^{*}$ with $S \backslash I=$ $\{x \in S \mid x \notin I\}$. Thus $S / I=(S \backslash I) \cup\{\overline{0}\}$, where $\overline{0}$ is the zero of $S / I$. Except when variations are noted in this paper, the terminology and notation is the same as that used in [1].

\section{Commutative $H I$ semigroups.}

THEOREM 1. If $S$ is a commutative HI semigroup, then $S$ is a group.

Proof. Assume that $S$ is not a group. Thus there is an $x \in S$ such that $x S \neq S$. Then $S \cong S / x S$ so $S$ contains a zero. We first show that under this assumption $S$ is nil.

Let $Z=\left\{x \in S \mid x y=0\right.$ for some $\left.y \in S^{*}\right\}$. Since $Z$ is an ideal of $S$ either $S \cong S / Z$ or $S=Z$. If $S \cong S / Z$, it follows that $Z=\{0\}$, so $S^{*}$ is a proper subsemigroup of $S$ and the map $\theta: S \rightarrow\{0,1\}$ which sends each element of $S^{*}$ onto 1 and sends 0 onto 0 is a homomorphism of $S$ onto the multiplicative semigroup $\{0,1\}$, a contradiction of the $H I$ property of $S$. Thus $S=Z$.

For a fixed element $a \in S^{*}$ define the set $A=\left\{x \in S \mid x a^{n}=0\right.$ for some postive integer $n\}$. Clearly $A$ is an ideal of $S$. If $a \notin A, a \in S / A$ and since $Z=S \cong S / A$, there is an element $b \in S \backslash A$ such that $a b \in A$, say $(a b) a^{n}=0$ or $b a^{n+1}=0$, so $b \in A$, a contradiction. Thus $a \in A$ and hence $a$ is nilpotent. Since $a$ was arbitrary it follows that $S$ is nil.

Let $x \in S$ such that $x \in x S$, say $x=x e$. Then $x=x e^{n}$ for each positive integer $n$. Since $S$ is nil, this implies that $x=0$. Thus, if $x \in S^{*}, \quad x \in S / x S$ and $S / x S \cong S$. But $x(S / x S)=\overline{0}$ so there exists $y \in S^{*}$ such that $y S=0$. Let $J=\{x \in S \mid x S=0\} . \quad J$ is an ideal of $S$ so either $J=S$ or $S \cong S / J$. If $S=J$, every nonempty subset of $S$ is an ideal of $S$ and thus $S \cong S / A$ for each $A \subseteq S, A \neq \varnothing$. Clearly this is not the case. By a similar argument, it follows that $S=S^{2}$. We now have $S \cong S / J$, so there is an $a \in S / J$ such that $a(S / J)=\overline{0}$, i.e., $a(S \backslash J) \subset J$, and hence $a S \subset J$. Therefore, $a S=$ $a S^{2} \subseteq J S=0$, so $a S=0$. But this contradicts the choice of $a$, and our proof is complete. 
COROLlaRY. If $S$ is a commutative $H I$ semigroup, then $S=$ $Z\left(p^{\infty}\right)$, for some prime $p$.

2. Inverse $H I$ semigroups containing a primitive idempotent. Following the notation of [1], p. 72, let $J(x)=S^{1} x S^{1}$ and $I(x)=$ $\{y \in J(x) \mid J(y) \neq J(x)\}$. It easily follows (see [1], p. 73) that if $J(x) \neq \varnothing$, $I(x)$ is an ideal of $S$ such that $J(x) / I(x)$ is either 0 -simple or the null semigroup of order 2.

Lemma 1. If $S$ is an inverse HI semigroup, either $S$ is simple or else $S=S^{\circ}$ and $S$ contains a unique 0-simple ideal $K$ contained in every nonzero ideal of $S$.

Proof. Suppose $S$ is not simple, say $a \in S$ such that $S \neq S a S=$ $S^{1} a S^{1}=J(a)$. Then $S \cong S / J(a)$, so $S$ contains a zero. It follows from the remark above that $J(a) / I(a)$ is 0 -simple. $S \cong S / I(a)$ implies $S$ contains a 0 -simple ideal $K \cong J(a) / I(a)$.

Let $U$ denote the union of all ideals $B$ of $S$ such that $K \cap B=0$. $U$ is a proper ideal of $S(K \neq 0)$ so $S \cong \bar{S}=S / U$. Since $K \cap U=0$, we can consider $K$ as an ideal of $\bar{S}$. It easily follows that every nonzero ideal of $\bar{S}$ has nonzero intersection with the 0 -simple ideal $K$. $S \cong \bar{S}$ implies the desired result.

We call this unique 0 -simple ideal the kernel of $S$.

Lemma 2. Let $S=S^{\circ}$ be an inverse semigroup and let $B$ be an ideal of $S$. Then $B$ is a Brandt subsemigroup of $S$ if and only if $B=S e S$ for some primitive idempotent $e \in S$.

Proof. Let $e$ be primitive in $S$ and let $I$ be an ideal of $S$ such that $I \subseteq S e S$. Suppose $e \notin I$ and let $f \in E_{I} \subseteq I \subseteq S e S$, say $f=a e b$. Then $a^{-1} f b^{-1}=h e$, where $h=a^{-1} a b b^{-1} \in E_{S}$. If $h e=e, e \in S f S \subseteq I$, contrary to our assumption. Therefore, by the primitivity of $e$, $h e=0$, so $f=a h e b=0$ and thus $E_{I}=\{0\}$. It follows that $I=\{0\}$. Clearly $(S e S)^{2}=S e S$, so $S e S$ is 0 -simple and hence Brandt.

Conversely, suppose $B$ is Brandt ideal of $S$ and let $e \in E_{B^{*}}$. Clearly $B=S e S$. If $f \in E_{S}$, since $e f \in B$ and $(e f) e=e f$, either $e f=0$ or $e f=e$, so $e$ is primitive in $S$.

Theorem 2. The Brandt semigroup $B=B(G ; \Lambda)$ is $H I$ if and only if $|G|=1$ and $A$ is infinite (i.e., if and only if $B$ is homomorphically simple and infinite).

Proof. By [5], $B(1 ; \Lambda)$ is a homomorph of $B$ and $|B(1 ; \Lambda)| \geqq 2$. 
Hence $B$ is $H I$ if and only if $B=B(1 ; \Lambda)$ where $\Lambda$ is infinite.

LEMmA 3. Let $S$ be an inverse $H I$ semigroup with Brandt kernel $K$. Then $K=B(1 ; \Lambda)$ for some index set $\Lambda$.

Proof. Let $\rho$ be a congruence relation on $K$ such that $|K| \rho \mid>1$. If $\rho^{\prime}$ denotes the identity extension of $\rho$ to all of $S\left(x \rho^{\prime} y\right.$ if and only if $x=y$ or $x \rho y$ ), it follows from [2] that $\rho^{\prime}$ is a congruence relation on $S$. Since $\left|S / \rho^{\prime}\right|>1, S \cong S / \rho^{\prime}$. Thus $K$, the unique Brandt kernel of $S$, is isomorphic to $K / \rho^{\prime}=K / \rho$, the unique Brandt kernel of $S / \rho^{\prime}$. It follows from Preston [5], that $B(G ; \Lambda) \cong B(G \theta ; \Lambda)$ and thus $G \cong G \theta$ for every homomorphism $\theta$ on $G$. Therefore $G=1$.

THEOREM 3. Let $S$ be an inverse HI semigroup containing a primitive idempotent $e$. Then $S$ satisfies one of the following:

(1) $S$ is an HI group,

(2) $S$ is an HI Brandt semigroup,

(3) $S$ has a transfinite composition series such that every factor is isomorphic to a (fixed) Brandt semigroup $B(1$; 1 ) for some index set $\Lambda$.

Proof. If $0 \notin S, S$ is simple so $S e S=S$. Therefore $E_{S}=E_{S e S}=$ $\{e\}$, so $S$ is a group.

Next assume $0 \in S$. If $S e S=S$, it follows from Lemma 2 that $S$ is Brandt, so suppose $S e S \neq S$. By Lemma 3, the kernel $K=$ $S e S \cong B(1 ; \Lambda)$ for some index set $\Lambda$. If $x \in S^{*}$, then $S \cong S / I(x)$ so $S / I(x)$ contains a unique kernel $\bar{K} \cong K$. By the remark at the beginning of this section, $J(x) / I(x)$ is Brandt and hence it is the Brandt kernel of $S / I(x)$; that is, the factor $J(x) / I(x)$ in the composition series of $S$ is isomorphic to $K$. Moreover, $S$ cannot contain a maximal proper ideal $A$ since this would imply $S \cong S / A$ is 0 -simple. Thus the composition series is infinite.

THEOREM 4. The ideals of an inverse HI semigroup $S$ containing a primitive idempotent are well ordered by inclusion such that for each proper ideal $A$ of $S$ there is a unique ideal $A^{\prime}$ of $S$ with the properties (1) $A \subset A^{\prime}$ and (2) $A \subset B$ implies $A^{\prime} \subseteq B$ for any ideal $B$ of $S$. We call $A^{\prime}$ the successor of $A$.

Proof. If $0 \notin S, S$ is simple and the theorem holds trivially, so assume $0 \in S$. Suppose $S$ has ideals $A$ and $B$ such that $A \nsubseteq B$ and $B \nsubseteq A$. Then $S \cong \bar{S}=S /(A \cap B)$, and $\bar{A}=A /(A \cap B)$ and $\bar{B}=$ $B /(A \cap B)$ are ideals of $\bar{S}$ such that $\bar{A} \cap \bar{B}=\overline{0}$, a contradiction of Lemma 1. Thus the ideals are linearly ordered by the inclusion relation. 
If $A$ is a proper ideal of $S, S \cong S / A$ so $S / A$ contains an ideal $\bar{K} \cong K$ (the Brandt kernel of $S$ ). Then $\bar{K}$ is of the form $A^{\prime} / A$ for some ideal $A^{\prime}$ of $S$. Since inclusion linearly orders the ideals, it follows that $A^{\prime}$ is unique. Clearly $A^{\prime}$ satisfies conditions (1) and (2) of the theorem.

Next let $\mathscr{A}$ denote a nonempty collection of ideals of $S$. Let $B=\cap\{A \mid A \in \mathscr{A}\}$, so either $B=S$ (and hence $B \in \mathscr{A}$ and $B$ is the least element of $\mathscr{A}$ ) or else $S \cong S / B$. Let $B^{\prime}$ denote the successor of $B$ and let $x \in B^{\prime} \backslash B$. It follows from the definition of $B$ that there is an ideal $A_{x} \in \mathscr{A}$ such that $x \notin A_{x}$. By Lemma 1 applied to $S / B \cong S$ we have $B \subseteq A_{x} \subset B^{\prime}$. Therefore, $B=A_{x} \in \mathscr{A}$ and $B$ is the least element of $\mathscr{A}$.

THEOREM 5. Let $S$ be an inverse semigroup containing a primitive idempotent $e$ such that $S$ is the union of the chain of ideals

$$
\{0\}=S_{0} \subset S_{1} \subset S_{2} \subset \cdots
$$

and such that for each $i \geqq 1$,

$$
S_{i} / S_{i-1} \cong B(1 ; \Lambda)
$$

where $\Lambda$ is some (fixed) index set. Then $S$ is HI if and only if for each $i \geqq 2$, there exist distinct idempotents $f, g_{1}$ and $g_{2}$ with $f \in S_{i} \backslash S_{i-1}$ and $g_{1}, g_{2} \in S_{i-1} \backslash S_{i-2}$ such that $g_{1}<f$ and $g_{2}<f$. Furthermore, if this is the case, then every idempotent of $S_{i} \backslash S_{i-1}$ has at least two nonzero idempotents under it.

Before proving the theorem, we introduce the following notation:

$$
B_{n}^{*}=S_{n} \backslash S_{n-1}, n \geqq 1 \text {. }
$$

Thus $S_{n}$ can be considered as the extension of $S_{n-1}$ by $B_{n}$. Note that $B_{1}=S_{1}$ is the kernel of $S$.

$$
B_{n}=B\left(1_{n} ; \Lambda\right)=\{(i, n, j) \mid i, j \in \Lambda\} \cup\left\{0_{n}\right\}
$$

where

$$
(i, n, j)\left(i^{\prime}, n, j^{\prime}\right)= \begin{cases}\left(i, n, j^{\prime}\right) & \text { if } j=i^{\prime} \\ 0_{n} & \text { otherwise } .\end{cases}
$$

Therefore, under the multiplication of $S$, if $j=i^{\prime}$, the above product remains the same, while if $j \neq i^{\prime}$, the above product lies in $S_{n-1}$.

For simplicity, we write

$$
e_{n, i}=(i, n, i) \text {. }
$$

Thus, the theorem asserts that $S$ is $H I$ if and only if for each $n \geqq 2$ 
there exists $i, r, s \in \Lambda$ such that

$$
e_{n, i}>e_{n-1, r} \text { and } e_{n, i}>e_{n-1, s} \text {, where } e_{n-1, r} \neq e_{n-1, s} \text {. }
$$

Proof of Theorem 5. First assume that $S$ is HI. It follows from Lemma 2 that for each $i \in \Lambda, e_{2, i}$ is not primitive in $S$ so there exists $v_{1}=v_{1}(i) \in \Lambda$ such that $e_{1, v_{1}}<e_{2, i}$. Assume inductively that for each $i \in \Lambda$, there exists $v_{n-1}=v_{n-1}(i) \in \Lambda$ such that $e_{n-1, v_{n-1}}<e_{n, 1}$. Since $S \cong S / S_{n-1}$, it follows that for each $i \in \Lambda$ there exists $v_{n} \in \Lambda$ such that $e_{n, v_{n}}<\boldsymbol{e}_{n+1, i}$.

Suppose that for each $i \in \Lambda, e_{2, i}$ has exactly one nonzero idempotent under it. Let $\rho$ be the congruence relation on $S$ generated by the relation $\rho_{0}=\left\{e_{2,1}, e_{1, v}\right\}$, where $e_{1, v}<e_{2,1}$. If $\rho$ is not one-to-one on $S_{1}$ it follows that $S_{2} / \rho=0$ so there exist $x, y \in S$ such that $x e_{2,1} y \neq 0$ and $x e_{1, v} y=0$. Therefore the idempotent $e=x^{-1} x e_{2,1} y y^{-1} \neq$ 0 . Since $e \leqq e_{2,1}$, it follows from our assumption that either $e=e_{2,1}$ or $e=e_{1, v}$. Thus in either case, we have

$$
e_{1, v}=e_{1, v} \cdot e=e_{1, v} x^{-1} x e_{2,1} y y^{-1}=x^{-1} x e_{1, v} y y^{-1}=0,
$$

a contradiction. Therefore, $\rho$ merely identifies corresponding terms of $B_{1}$ and $B_{2}$. Relabeling if necessary, we have $e_{1, j}<e_{2, j}$ for each $j$ in $\Lambda$, and by induction $e_{n, j}<e_{n+1, j}, n \geqq 1, j \in \Lambda$. Define $\sigma$ to be the congruence relation on $S$ generated by the relation

$$
\sigma_{0}=\left\{\left(e_{n, i}, e_{m, i}\right) \mid n, m \geqq 1, i \in \Lambda\right\} .
$$

Clearly $\sigma$ is one-to-one on $S_{1}$, and since $S / \sigma$ has no proper nonzero ideals, we cannot have $S \cong S / \sigma$, a contradiction.

To prove the sufficiency of the condition let $S$ be an inverse semigroup of the type described in the theorem and suppose that for each $n \geqq 1$ there exist distinct $a(n)$ and $b(n)$ in $\Lambda$ such that

$$
e_{n, a(n)}<e_{n+1,1} \text { and } e_{n, b(n)}<e_{n+1,1},
$$

and let $\tau$ be a congruence relation on $S$ that is not one-to-one. Since $x \tau y$ implies $x x^{-1} \tau y y^{-1}$ and $x^{-1} x \tau y^{-1} y$, it follows from the structure of $S$ that $\tau$ is not one-to-one on $E_{S}$.

If $e_{n, u} \tau e_{n, v}, u \neq v$, then $e_{n, u} \tau e_{n, u} e_{n, v}$ so that we may assume without loss of generality that there exist integers $n$ and $m$ with $n>m$ and $r, s \in \Lambda$ such that

$$
e_{n, r} \tau e_{m, s}
$$

Then

$$
(1, n, r) e_{n, r}(r, n, 1) \tau(1, n, r) e_{m, s}(r, n, 1) \text {. }
$$

Upon multiplying both sides of (2) by $e_{n-1, a}$ we obtain the relation 
$e_{n-1, a} \tau e_{r, s}$ for some $r<n-1$ and some $s \in \Lambda$. As above, this implies

$$
e_{n-1,1} \tau e_{u_{2}, v_{2}}, \text { where } u_{2}<n-1 \text {. }
$$

(If $e_{u_{1}, v_{1}}=e_{n-1, a}$ multiply both sides of (2) by $e_{n-1, b}$.)

Continuing in this manner we conclude that $e_{1,1} \tau 0$ and thus by the transitivity of $\tau$ we conclude that $\left|S_{n} / \tau\right|=1$. If there exists an integer $N$ such that $\tau$ is not one-to-one on $S_{N}$ but is one-to-one on $S / S_{N}$, then $S / \tau=S / S_{N} \cong S$. If no such integer $N$ exists, $|S / \tau|=$ 1. Hence $S$ is $H I$.

The final assertion will follow if, when $S$ is $H I$, for each $i \in \Lambda$, there exist $r, s \in \Lambda, r \neq s$, such that

$$
e_{2, i}>e_{1, r} \text { and } e_{2, i}>e_{1, s}
$$

Without loss of generality assume $e_{2,1}>e_{1,1}$ and $e_{2,1}>e_{1,2}$. For each $i \in \Lambda$,

$$
(i, 2,1)(1,1,1)=\left(a_{i}, 1,1\right) \text { for some } a_{i} \in \Lambda \text {, }
$$

and

$$
(i, 2,1)(2,1,2)=\left(b_{i}, 1,2\right) \text { for some } b_{i} \in \Lambda
$$

Therefore

$$
(i, 2,1)(1,1,1)(1,2, i)=e_{1, a_{i}}
$$

and

$$
(i, 2,1)(2,1,2)(1,2, i)=e_{1, b_{i}} .
$$

Clearly $e_{1, a_{i}}<e_{2, i}$ and $e_{1, b_{i}}<e_{2, i}$. Furthermore,

$$
\begin{aligned}
e_{1, a_{i}} e_{1, b_{i}} & =(i, 2,1) e_{1,1}(1,2, i)(i, 2,1) e_{1,2}(i, 2, i) \\
& =(1,2,1) e_{1,1} e_{2,1} e_{1,2}(1,2, i)=(i, 2,1) e_{1,2} e_{1,1}(1,2, i)=0 .
\end{aligned}
$$

Therefore, $a_{i} \neq b_{i}$, and the proof is complete.

We conclude with an example of an $H I$ inverse semigroup of the type described in Theorem 5. Let $N$ denote the set of positive integers, and let $\left\{B_{n} \mid n \in N\right\}$ be a collection of pairwise disjoint isomorphic copies of the Brandt semigroup $B(1, N)$. As in Theorem 5 , write the nonzero elements of $B_{n}$ in the form $(i, n, j)$, for $i, j \in N$, let $0_{n}$ denote the zero of $B_{n}$, and write 0 for $0_{1}$.

Let $S_{1}=B_{1}$ and let $S_{n+1}$ be the extension of $S_{n}$ by $B_{n+1}$ where multiplication is defined as follows:

$$
\text { If } \alpha, \beta \in S_{n}, \alpha \circ \beta=\alpha \beta \text {. }
$$




$$
\text { If } \alpha, \beta \in B_{n+1}^{*}, \alpha \circ \beta=\left\{\begin{array}{ll}
\alpha \beta & \text { if } \alpha \beta \neq 0_{n+1} \\
0 & \text { if } \alpha \beta=0_{n+1}
\end{array}\right. \text {. }
$$

Products between $B_{n+1}^{*}$ and $S_{n}$ are defined recursively as follows:

$$
\begin{aligned}
& (i, n, j) \circ(r, n-1, s)= \begin{cases}(2 i-1, n-1, s) & \text { if } r=2 j-1, \\
(2 i, n-1, s) & \text { if } r=2 j, \\
0 & \text { otherwise . }\end{cases} \\
& (s, n-1, r) \circ(j, n, i)=[(i, n, j) \circ(r, n-1, s)]^{-1} \\
& (i, n, j) \circ(r, n-k-1, s)=[(i, n, j) \circ(f(r), n-k, f(r))] \circ \\
& (r, n-k-1, s) \text {, }
\end{aligned}
$$

where $f(r)$ is the greatest integer less than or equal to $\frac{r+1}{2}$.

$$
\begin{gathered}
(s, n-k-1, r) \circ(j, n, i)=[(i, n, j) \circ(r, n-k-1, s)]^{-1} \\
(i, n, j) \circ 0=0 \circ(i, n, j)=0 .
\end{gathered}
$$
follows:

Defining $S=\mathbf{U}_{n \geq 1} S_{n}$, it can be shown that $S$ is a semigroup as

Let $\phi_{1}=(i, n, j), \phi_{2}=(r, m, s)$ and $\phi_{3}=(u, p, v)$. First observe that $\left(\phi_{1} \phi_{2}\right) \phi_{3}=\phi_{1}\left(\phi_{2} \phi_{3}\right)$ if $|n-m| \leqq 1$ and $|m-p| \leqq 1$. Because of the way multiplication is defined it is sufficient to consider the following cases to show this: (i) $m=n, p=n-1$; (ii) $m=p=$ $n-1$; (iii) $m=n-1, p=n$; (iv) $m=n+1, p=n$; (v) $m=n-1$, $p=n-2$. Associativity can be shown in each of the above cases by direct computation. Clearly this can be generalized to show that any product where consecutive factors come from $B_{i}^{*} \cup B_{j}^{*}$, with $|i-j| \leqq 1$, can be associated in any manner.

Next, observe that $e_{n, r}<e_{n+1, f(r)}<e_{n+2, f^{2}(r)}<\cdots$, where $f^{k+1}(r)=$ $f\left(f^{k}(r)\right)$. Thus every product in $S$ can be written as a product where consecutive factors are of the form $(i, n, j) \circ(r, m, s)$ such that $|n-m| \leqq 1$. Therefore, applying the observation made above, we see that $S$ is a semigroup. Furthermore,

$$
e_{n, 1}<e_{n+1,1} \text { and } e_{n, 2}<e_{n+1,1} \text { for each } n \geqq 1 \text {, }
$$

so by Theorem $4, S$ is $H I$.

The following example illustrates the associativity of $S$ :

Let $\phi_{1}=(3, n, 2), \phi_{2}=(3, n-1,2)$ and $\phi_{3}=(3, n-2,2)$. Then

$$
\left(\phi_{1} \phi_{2}\right) \phi_{3}=(5, n-1,2) \phi_{3}=(9, n-2,2)
$$

and

$$
\begin{aligned}
\phi_{1}\left(\phi_{2} \phi_{3}\right) & =\phi_{1}(5, n-2,2)=\left[\phi_{1}(3, n-1,3)\right](5, n-2,[2) \\
& =(5, n-1,3)(5, n-2,2)=(9, n-2,2) .
\end{aligned}
$$


The author wishes to thank the referee for his helpful suggestions which led to Theorem 4.

\section{REFERENCES}

1. A. H. Clifford and G. B. Preston, The Algebraic theory of semigroups, Vol. I, Amer. Math. Soc., Providence, Rhode Island, 1961.

2. B. A. Jensen, Inverse semigroups that are homomorphically finite (to appear)

3. B. A. Jensen and D. W. Miller, Commutative semigroups that are almost finite, Pacific J. Math. 27 (1968), 533-538.

4. I. Kaplansky, Infinite Abelian groups, The University of Michigan Press, Ann Arbor, Michigan, 1954.

5. G. B. Preston, Congruences of Brandt semigroups, Math. Ann. 139 (1959), 91-94.

Received October 25, 1967.

Portland State University 



\section{PACIFIC JOURNAL OF MATHEMATICS}

\section{EDITORS}

H. ROYDEN

Stanford University

Stanford, California

\author{
R. R. Phelps \\ University of Washington \\ Seattle, Washington 98105
}

J. DugundJI

Department of Mathematics

University of Southern California

Los Angeles, California 90007

RICHARD ARENS

University of California

Los Angeles, California 90024

\section{ASSOCIATE EDITORS}

E. F. BECKenbaCH

B. H. NEUMANN

F. WOLF

K. YoSHIDA

\section{SUPPORTING INSTITUTIONS}

\author{
UNIVERSITY OF BRITISH COLUMBIA \\ CALIFORNIA INSTITUTE OF TECHNOLOGY \\ UNIVERSITY OF CALIFORNIA \\ MONTANA STATE UNIVERSITY \\ UNIVERSITY OF NEVADA \\ NEW MEXICO STATE UNIVERSITY \\ OREGON STATE UNIVERSITY \\ UNIVERSITY OF OREGON \\ OSAKA UNIVERSITY \\ UNIVERSITY OF SOUTHERN CALIFORNIA
}

\author{
STANFORD UNIVERSITY \\ UNIVERSITY OF TOKYO \\ UNIVERSITY OF UTAH \\ WASHINGTON STATE UNIVERSITY \\ UNIVERSITY OF WASHINGTON \\ $\stackrel{*}{*} \stackrel{*}{*} \stackrel{*}{*}$ AMERICAN MATHEMATICAL SOCIETY \\ CHEVRON RESEARCH CORPORATION \\ TRW SYSTEMS \\ NAVAL WEAPONS CENTER
}

The Supporting Institutions listed above contribute to the cost of publication of this Journal, but they are not owners or publishers and have no responsibility for its content or policies.

Mathematical papers intended for publication in the Pacific Journal of Mathematics should be in typed form or offset-reproduced, double spaced with large margins. Underline Greek letters in red, German in green, and script in blue. The first paragraph or two must be capable of being used separately as a synopsis of the entire paper. It should not contain references to the bibliography. Manuscripts, in duplicate if possible, may be sent to any one of the four editors. Please classify according to the scheme of Math. Rev. 36, 1539-1546. All other communications to the editors should be addressed to the managing editor, Richard Arens, University of California, Los Angeles, California, 90024.

50 reprints are provided free for each article; additional copies may be obtained at cost in multiples of 50 .

The Pacific Journal of Mathematics is published monthly. Effective with Volume 16 the price per volume (3 numbers) is $\$ 8.00$; single issues, $\$ 3.00$. Special price for current issues to individual faculty members of supporting institutions and to individual members of the American Mathematical Society: $\$ 4.00$ per volume; single issues $\$ 1.50$. Back numbers are available.

Subscriptions, orders for back numbers, and changes of address should be sent to Pacific Journal of Mathematics, 103 Highland Boulevard, Berkeley, California, 94708.

PUBLISHED BY PACIFIC JOURNAL OF MATHEMATICS, A NON-PROFIT CORPORATION

Printed at Kokusai Bunken Insatsusha (International Academic Printing Co., Ltd.), 7-17, Fujimi 2-chome, Chiyoda-ku, Tokyo, Japan. 


\section{Pacific Journal of Mathematics \\ Vol. 29, No. $3 \quad$ July, 1969}

Herbert James Alexander, Extending bounded holomorphic functions from certain subvarieties of a polydisc ...................... 485

Edward T. Cline, On an embedding property of generalized Carter

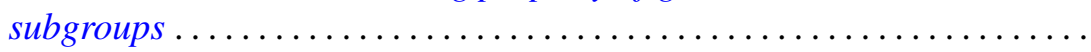

Roger Cuppens, On the decomposition of infinitely divisible characteristic functions with continuous Poisson spectrum. II ...............

William Richard Emerson, Translation kernels on discrete Abelian

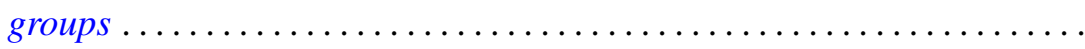

Robert William Gilmer, Jr., Power series rings over a Krull domain ....... 543

Julien O. Hennefeld, The Arens products and an imbedding theorem ...... 551

James Secord Howland, Embedded eigenvalues and virtual poles ........ 565

Bruce Ansgar Jensen, Infinite semigroups whose non-trivial homomorphs are all isomorphic .............................. 583

Michael Joseph Kascic, Jr., Polynomials in linear relations. II .......... 593

J. Gopala Krishna, Maximum term of a power series in one and several

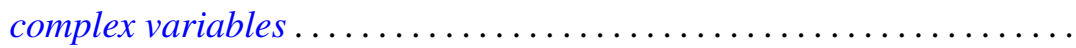

Renu Chakravarti Laskar, Eigenvalues of the adjacency matrix of cubic lattice graphs ...................................

Thomas Anthony Mc Cullough, Rational approximation on certain plane

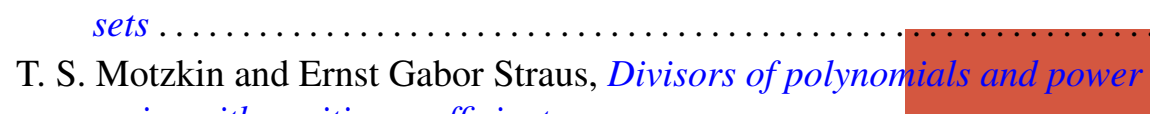
series with positive coefficients .

Graciano de Oliveira, Matrices with prescribed characteristic polynomial and a prescribed submatrix.

Graciano de Oliveira, Matrices with prescribed characteristic polynomial and a prescribed submatrix. II .

Donald Steven Passman, Exceptional 3/2-transitive permutation groups .................................

Grigorios Tsagas, A special deformation of the metric with no negative sectional curvature of a Riemannian space............

Joseph Zaks, Trivially extending decompositions of $E^{n}$ 